Provided for non-commercial research and education use. Not for reproduction, distribution or commercial use.

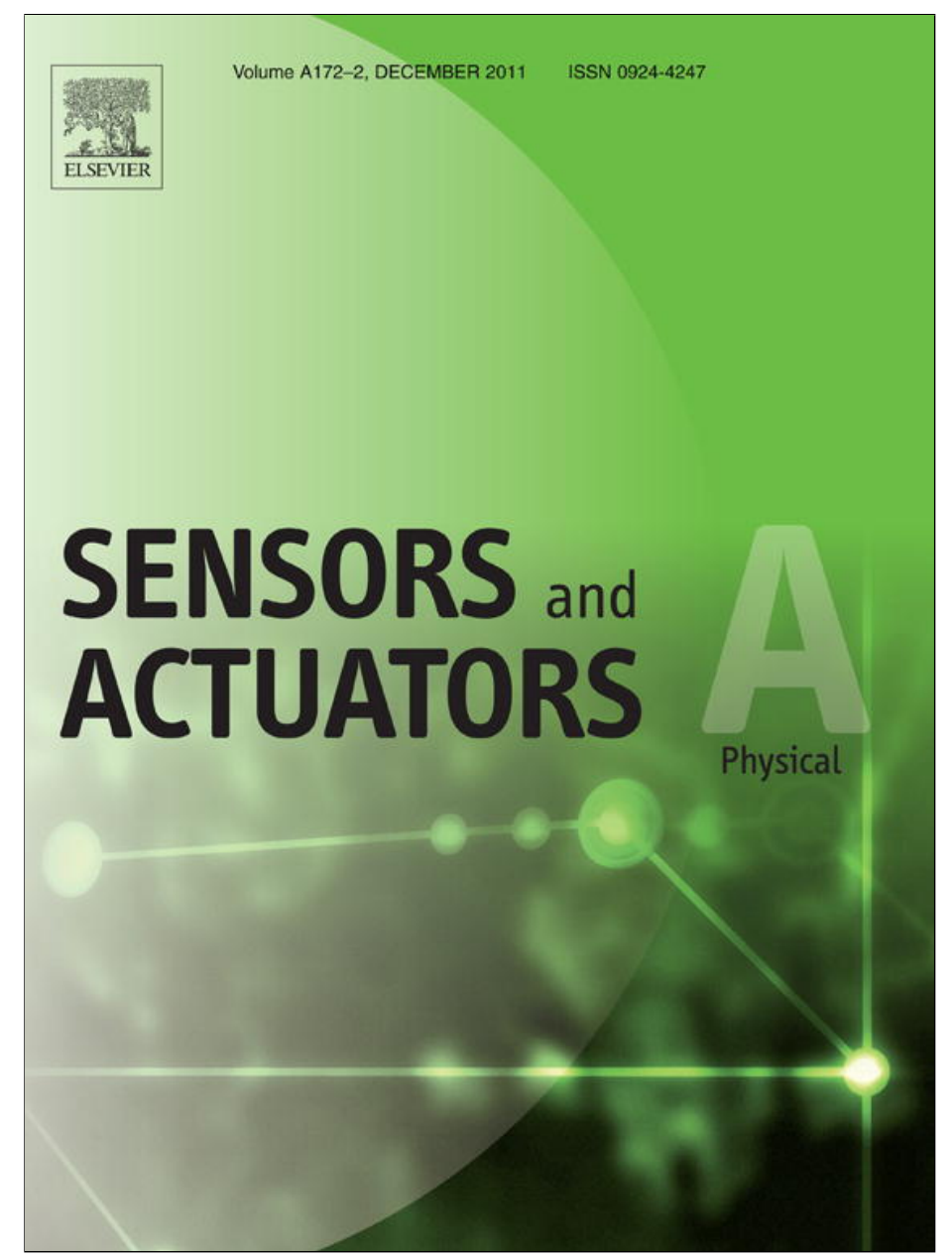

This article appeared in a journal published by Elsevier. The attached copy is furnished to the author for internal non-commercial research and education use, including for instruction at the authors institution and sharing with colleagues.

Other uses, including reproduction and distribution, or selling or licensing copies, or posting to personal, institutional or third party websites are prohibited.

In most cases authors are permitted to post their version of the article (e.g. in Word or Tex form) to their personal website or institutional repository. Authors requiring further information regarding Elsevier's archiving and manuscript policies are encouraged to visit:

http://www.elsevier.com/copyright 


\title{
A comparison study of pattern recognition algorithms implemented on a microcontroller for use in an electronic tongue for monitoring drinking waters
}

\author{
Eduardo Garcia-Breijo ${ }^{\mathrm{a}, *}$, John Atkinson ${ }^{\mathrm{b}}$, Luis Gil-Sanchez ${ }^{\mathrm{a}}$, Rafael Masot ${ }^{\mathrm{a}}$, Javier Ibañez ${ }^{\mathrm{a}}$, \\ Jose Garrigues ${ }^{\mathrm{a}}$, Monika Glanc ${ }^{\mathrm{b}}$, Nicolas Laguarda-Miro ${ }^{\mathrm{a}}$, Cristian Olguin ${ }^{\mathrm{a}}$ \\ a Centro de Reconocimiento Molecular y Desarrollo Tecnológico, Unidad Mixta UPV-UV Universitat Politècnica de Valéncia, Camino de Vera s/n, 46022 Valencia, Spain \\ ${ }^{\mathrm{b}}$ Faculty of Engineering and Environment, University of Southampton, Southampton SO17 1BJ, UK
}

\section{A R T I C L E I N F O}

\section{Article history:}

Received 26 April 2011

Received in revised form 17 June 2011

Accepted 29 September 2011

Available online 10 October 2011

\section{Keywords:}

Electronic tongue

Pattern recognition

Neural network

Thick-film

Microcontroller

\begin{abstract}
A B S T R A C T
A portable electronic tongue has been developed using an array of eighteen thick-film electrodes of different materials forming a multi-electrode array. A microcontroller is used to implement the pattern recognition. The classification of drinking waters is carried out by a Microchip PIC18F4550 microcontroller and is based on neural networks algorithms. These algorithm are initially trained with the multi-electrode array on a Personal Computer (PC) using several samples of waters (still, sparkling and tap) to obtain the optimum architecture of the networks. Once it is trained, the computed data are programmed into the microcontroller, which then gives the water classification directly for new unknown water samples. A comparative study between a Fuzzy ARTMAP, a Multi-Layer Feed-Forward network (MLFF) and a Linear Discriminant Analysis (LDA) has been done in order to obtain the best implementation on a microcontroller.
\end{abstract}

(c) 2011 Elsevier B.V. All rights reserved.

\section{Introduction}

Sensors based on electrochemical techniques are used to determine the concentration of specific chemical compounds, or the accurate measurement of physiochemical parameters. But generally they have an important drawback; namely that of susceptibility to interference from other species that mask the species of interest. However this drawback can be converted to advantage if, instead of looking for that type of accurate measurement, another kind 7 measurement of a rather more qualitative nature is employed, such as the discrimination or classification of samples of complex chemical nature. Under this concept, electronic tongue systems that employ different sets of non-specific electrodes were developed some years ago [1]. Each of the electrodes provides a signal that is proportional to the set of species in the system under analysis. As electronic tongue systems tend to produce a qualitative result, multivariate analysis techniques are generally required in order to process the data obtained from the measurements.

Various electrochemical techniques have been used in electronic tongues, such as potentiometry [2], voltammetry [3] or impedance spectroscopy [4]. These have been used in several applications, including waste water control [5] and food analysis [6].

\footnotetext{
* Corresponding author. Tel.: +34 963877608; fax: +34 963877609.

E-mail address: egarciab@eln.upv.es (E. Garcia-Breijo).
}

Potentiometric techniques have as their main desirable feature simplicity of measurement method and electronic equipment. Various different types of electrodes have been used in potentiometry, such as membranes [7] or metal surfaces [8]. In this latter electrode, a voltage is obtained that is proportional to the concentrations of all species present in solution and hence its quantification is difficult to determine whenever the aqueous medium is complex [9].

A method for obtaining a multi-electrode of easy construction and simple operation is to employ inks from thick-film hybrid circuit technology [10] because there are many different types of inks and each has a key chemical element that can become the active element of the sensor.

Most systems of electronic tongues remain in the laboratory version, which requires the presence of a computer and, specially above all, two separate processes, one for taking measurements and another for data processing. If it is desired for these systems to have industrial application however, it is necessary to unify these two phases into a single system. The best method for achieving a single system is the use of microcontrollers in systems which, in addition to the measurement of potential, are able to perform the analysis of relevant data using a software program implemented in the microcontroller memory. Thus portable electronic tongues are becoming popular as they offer simplicity, reliability and use in field [11]. Some systems using microprocessors have been presented as electronic tongues [12] but the system presented in this communication has as its main novelty the development and comparison of three types of pattern recognition algorithms. Pattern 
recognition algorithms have become a critical component in the implementation of electronic tongues and noses and have been used successfully in these applications [13]. For implementation in portable equipment the algorithm must be transferable to a microcontroller which has a limited amount of memory. Thus the perfect pattern recognition algorithms will require high accuracy, to work fast to work in real-time and have low memory requirements in order to be implemented in a microcontroller. Not all pattern recognition algorithms are able to reach each of these requirements. In this communication three pattern recognition algorithms have been used, Fuzzy ARTMAP, Multi-Layer Feed-Forward (MLFF) and Linear Discriminant Analysis (LDA).

MLFF is the most popular type of artificial neural network (ANN); basically it is formed basically by three layers of neurons (input, hidden and output). They require a training stage, where the weights of each neuron are set, and another validation stage [14]. The Fuzzy ARTMAP network uses the so-called adaptive resonance method and is based on the use of prior actions to predict subsequent steps [15]. For LDA the method is a probabilistic parametric classification technique and maximizes the variance between categories and minimizes the variance within categories, by means of a data projection from a high dimensional space to a low dimensional space. In this way, a number of orthogonal linear discriminant functions equal to the number of categories minus one are obtained $[16,17]$. Such algorithms have been used in electronic noses [18] and electronic tongue systems [19] giving important benefits such as: simplicity of implementation of computer algorithm, speed of calculation and the attainment of good and reliable results with a small number of measures.

The aim of this paper is to present a potentiometric electronic tongue system that uses an electrode assembly constructed in thick film technology whose data analysis system consists of a pattern recognition algorithm implemented on a microprocessor system. As an example application of this system, an analysis has been made of various types of drinking water that have different concentrations and types of salts. The implemented pattern recognition algorithm is able to perform a classification of these water samples using the data obtained from potentiometric measurements. This example can be extended to other industrial applications such as quality control of water purification, wastewater discharges, quality control of drinks and, in general, in cases where it is appropriate to conduct qualitative measures quickly, easily, economically, and not necessarily carried out by specialized personnel.

\section{System description}

\subsection{Samples}

A total of five Spanish natural mineral waters of different brands (Bezoya, Bronchales, Cortes, Lanjarón and Solán), one sparkling water (Primavera) and tap water from Valencia City have been selected as representative samples and they have been studied by using the array of electrodes described below. The names and concentrations (in $\mathrm{mg} / \mathrm{L}$ ) of the main ions for the used mineral waters are listed in Table 1.

\subsection{Electrodes}

A wide range of electrodes with different surfaces were selected in order to explore their differential response in potentiometric measurements. Following this approach various electrodes fabricated using thick-film technology were prepared. To this purpose, several inks with different active element were used; the pastes were supplied by HERAEUS and they are $\mathrm{RuO}_{2}$ of $10 \Omega$ /sq (model R8911) and $1 \mathrm{M} \Omega / \mathrm{sq}$ (model R8961), Cu (model C7257), Ag (model

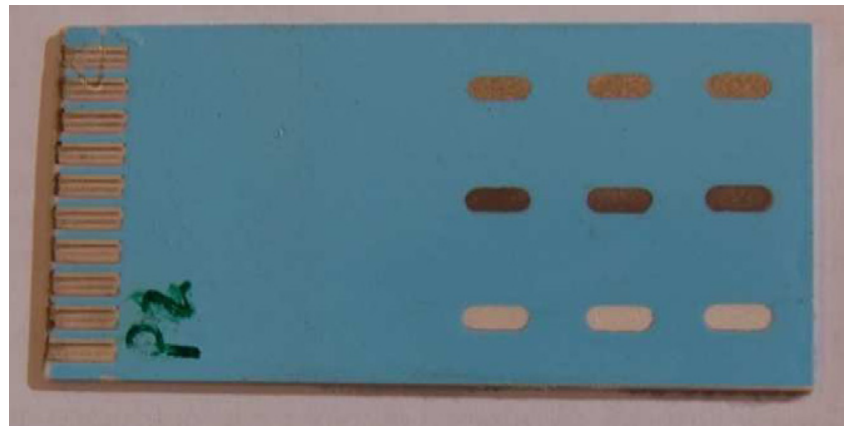

Fig. 1. Top side of multi-electrode realized in thick-film technology.

C8829), and Pt (model C1076D). The $\mathrm{AgCl}$ was manufactured by mixing $\mathrm{Ag}$ and $\mathrm{AgCl}$ powder in a ratio of $1: 1$ and using low temperature EG2020 glass (supplied by Ferro). The protective upper layer paste was model D2020823D2 supplied by GWENT. The process of fabricating these types of electrodes has been explained in a previous paper $[15,20]$.

The electrodes were supported on an alumina substrate RUBALIT 708S (supplied by Ceramic Tec) with an area of $50.8 \mathrm{~mm} \times 25.4 \mathrm{~mm}$ and thickness of $0.635 \mathrm{~mm}$. In order to prepare the above mentioned set of electrodes, three thick film printer screens were made, corresponding to three layers: namely, the conductive layer working as an electronic interconnect for the signal, the active layer and the upper protection layer. The conductive paste used was Ag C8829 (supplied by HERAEUS). The layout of the tracks was designed to join the ceramic substrate to a flat cable connector with a separation of $3 \mathrm{~mm}$ between terminals.

Three different electrodes of each type were simultaneously employed $\left(\mathrm{Cu}, \mathrm{RuO}_{2}\right.$ of $10 \Omega / \mathrm{sq}, \mathrm{RuO}_{2}$ of $1 \mathrm{M} \Omega / \mathrm{sq}, \mathrm{Ag}, \mathrm{Pt}$ and $\mathrm{AgCl})$. Thus we obtain a set of 18 electrodes, which was used as an active system for potentiometric measurements forming a multi-electrode board (Fig. 1 shows the final array of electrodes implemented in thick-film technology). Using 6 electrodes prepared from the same inks, the quantity of electrodes was enough to be able to study the respond of each material and moreover to obtain the respond average.

\subsection{Electronic system}

Two boards with 18 electrodes on each were used. Therefore 36 channels could be measured simultaneously. The external reference electrode employed was an $\mathrm{Ag} / \mathrm{AgCl}$ device (supplied by CRISON).

Measurements were carried out using an own design portable data logger. The output signals of the multi-electrode were acquired using a 36:1 multiplexer architecture, which was formed by two 18:1 channel MOS analog multiplexers (MAX306, MAXIM) and one 8:1 channel analog multiplexer (MAX308, MAXIM). The selection of each channel in the multiplexer was controlled by the microcontroller. The sampling rate for the 36 channels was one electrode every $100 \mathrm{~ms}$ in periods of $10 \mathrm{~s}$.

A precision CMOS quad micro power Operational Amplifier (LMC646, NATIONAL SMC), was connected to the output multiplexer. This operational amplifier (AO) has very high input impedance (ultra low input bias current of less than $16 \mathrm{fA}$ ) and hence is suited to the signal impedance generated by the potentiometric multi-electrode.

An analog to digital converter (A/D) (MAX128, MAXIM) has been used because the AD of the microcontroller is merely of 10 bits resolution and it only accepts positive voltage. This A/D has a resolution of 12-bits and can work with unipolar or bipolar input signals. It uses an external or internal reference voltage in order to obtain 
Table 1

Concentration (in $\mathrm{mg} / \mathrm{L}$ ) of certain anions and cations in mineral waters according to information provided by manufactures.

\begin{tabular}{|c|c|c|c|c|c|c|c|c|c|}
\hline Water type & $\mathrm{CO}_{3} \mathrm{H}^{-}$ & $\mathrm{SO}_{4}^{-}$ & $\mathrm{Cl}^{-}$ & $\mathrm{Ca}^{2+}$ & $\mathrm{SiO}_{2}$ & $\mathrm{Mg}^{2+}$ & $\mathrm{Na}^{+}$ & $\mathrm{F}^{-}$ & $\mathrm{K}^{+}$ \\
\hline Bezoya & 18 & 15 & 0.67 & 4 & 8.9 & 1.8 & 1 & - & - \\
\hline Bronchales & 19 & 6 & 2 & 3 & 9 & 3 & 1.35 & 0.1 & 1.36 \\
\hline Cortes & 268.4 & 17.6 & 8.7 & 81.9 & 9.2 & 37 & 6.4 & 1 & 0.7 \\
\hline Tap water & 154 & 258 & 84 & 117 & 3.3 & 37 & 49 & - & 2.5 \\
\hline Lanjarón & 105 & 17.3 & 2.8 & 27.2 & 4.8 & 8.8 & 4.8 & 0.2 & 1.1 \\
\hline Sparkly water & 297.2 & 43.9 & 35.8 & 88.7 & 7.1 & 23.4 & 20.7 & 0.1 & 1.3 \\
\hline Solan & 279.4 & 18 & 8.1 & 59.3 & 7.1 & 25.8 & 5.2 & 0.2 & 1.1 \\
\hline
\end{tabular}

different full scale ranges. In this case a $2.5 \mathrm{~V}$ external reference and a bipolar input signal were used. With this configuration the resolution (equivalent to 1 Least Significant Bit) is $1.22 \mathrm{mV}$. The PIC18F4550 microcontroller gathered the data from the A/D converter using an I2C bus. PIC18F4550 was selected [21] for its low power consumption (sleep mode currents down to $0.1 \mu$ A typical), $32 \mathrm{~K}$ of memory program and $2 \mathrm{~K}$ of RAM and USB port.

The software for the PIC18F4550 microcontroller has been designed to obtain the average value for each channel. Six input vectors are calculated using the 36 channels of data. These six input vectors correspond to the six types of electrodes.

The process of measurement has been divided in two stages: the training period and the test period. In the training period, the data were sent to the PC via an RS232 serial communications link in order to use them in the training algorithm with MATLAB ${ }^{\circledR}$ R2010b. The acquisition software was developed using Visual Basic ${ }^{\circledR} 6.0$ and Microsoft Excel ${ }^{\circledR} 2003$ software. In the test period, the data were measured and they were stored directly into the microcontroller in order to be used in the embedded neural network. A block diagram of the measurement system is shown in Fig. 2.

\subsection{Measurement process}

Initially the set of 36 electrodes were dipped at $25^{\circ} \mathrm{C}$ in $300 \mathrm{~mL}$ of $0.01 \mathrm{M} \mathrm{KNO}_{3}$ reference solution. The responses of the electrodes were studied every other day until they reached a stable potential, which happened after fifteen days. This stage was called the conditioning period (Fig. 3). Artificial neural networks training was carried out with the first samples until obtaining an acceptable recognition percentage (more than $80 \%$ ) as we can see in Section 3. That happened with the 8th sample (around 15 days).

After this initial period, measures were acquired every other day for the duration of a further forty days, using four bottles of each type of water. This stage was called the training and test period (Fig. 3). The groups of electrodes were immersed in the corresponding aqueous sample over a period of $10 \mathrm{~min}$ during the last $5 \mathrm{~min}$ of which their potentials were recorded. Typical time to steady state was always less than $5 \mathrm{~min}$. The measurements were performed at $25^{\circ} \mathrm{C}$. After each measurement, the electrodes were cleaned with distilled water. The samples were measured in a random order. After each measurement, the set of electrodes was again dipped in the reference solution. The response of the multi-electrode array was considered stable over the first 8 samples (see response to tap water sample in Fig. 4). Considering that the results obtained using the potentiometric measurement do not contribute to a clear discrimination between the samples, it is necessary to study them further using artificial neural networks.

\section{Data analysis}

The procedure for working with artificial neural networks consists of two stages, a first stage of training of the network and a second stage for its verification. The training stage is performed with some of the available measures. At this stage the network categories are set out (in our case the seven different types of water). The data form six electrodes for each measurement are applied as an input vector. With these data the coefficients of the algorithm that configures the network are calculated. In the verification stage, the data from new measures are applied to the inputs, checking whether the output of the active network is correct or not.

The program Matlab $2010 b^{\circledR}$ running on a PC computer has been used to train the networks. The computer to be used is determined by its computing power and ease of implementing the algorithms of the neural networks. By contrast, the verification stage is performed entirely in a microcontroller. To this end, the results obtained in the training stage are used as the coefficients of the algorithms that are incorporated into the microcontroller program. Through this way of working, once the training stage has been accomplished, the developed system can work independently of a PC. This is one of the key features of the equipment presented in this paper.

\subsection{Training the Fuzzy ARTMAP}

Fuzzy ARTMAP neural networks are based on the so-called adaptive resonance theory (ART) which aims to create algorithms that are adaptable to a significant response and remain stable in the

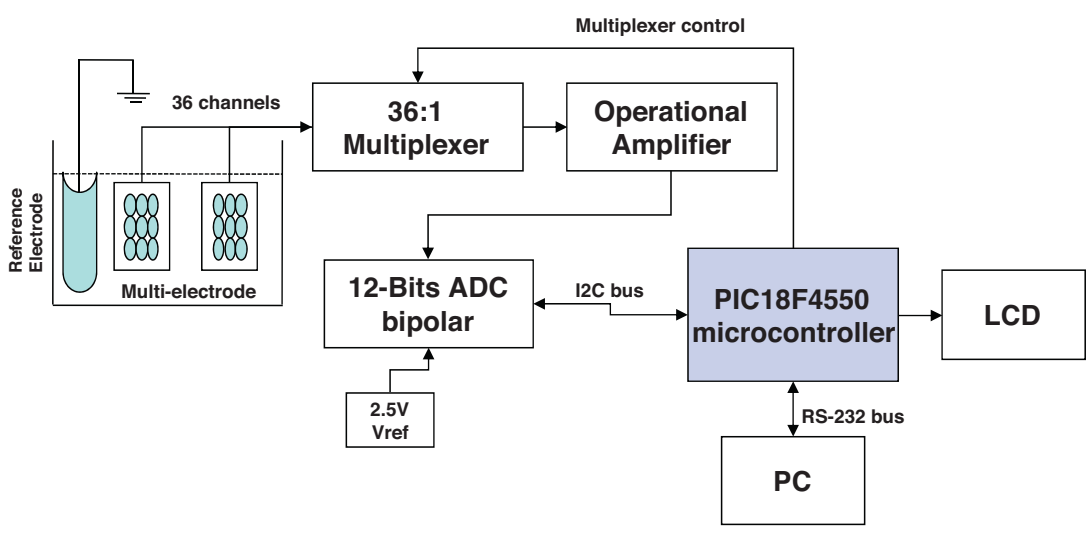

Fig. 2. Block diagram of the measurement system. 


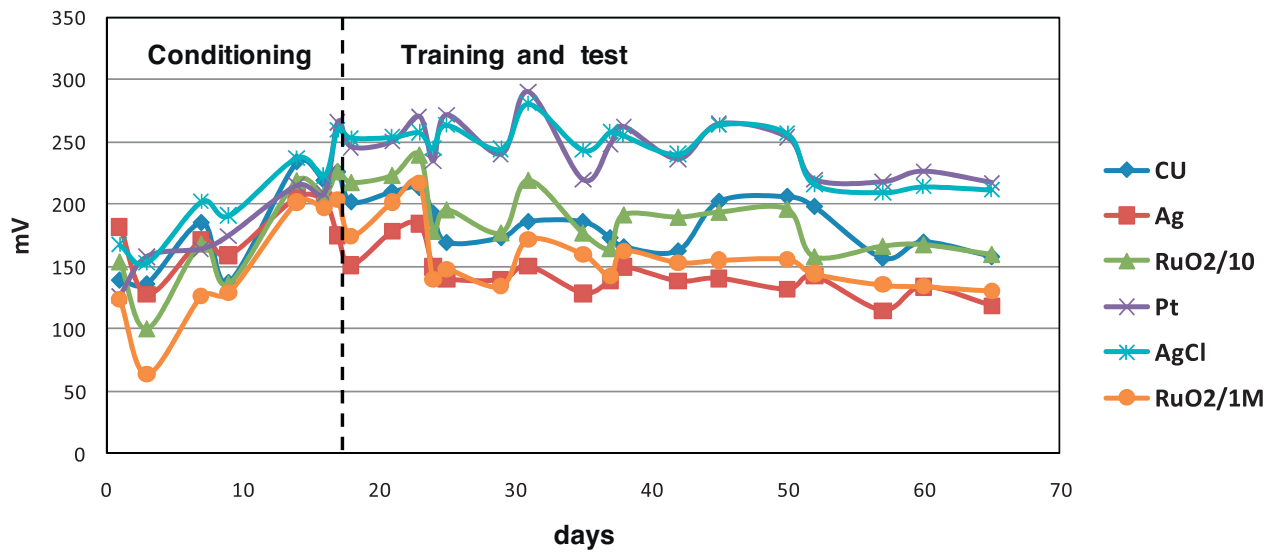

Fig. 3. Electrode responses in reference solution.

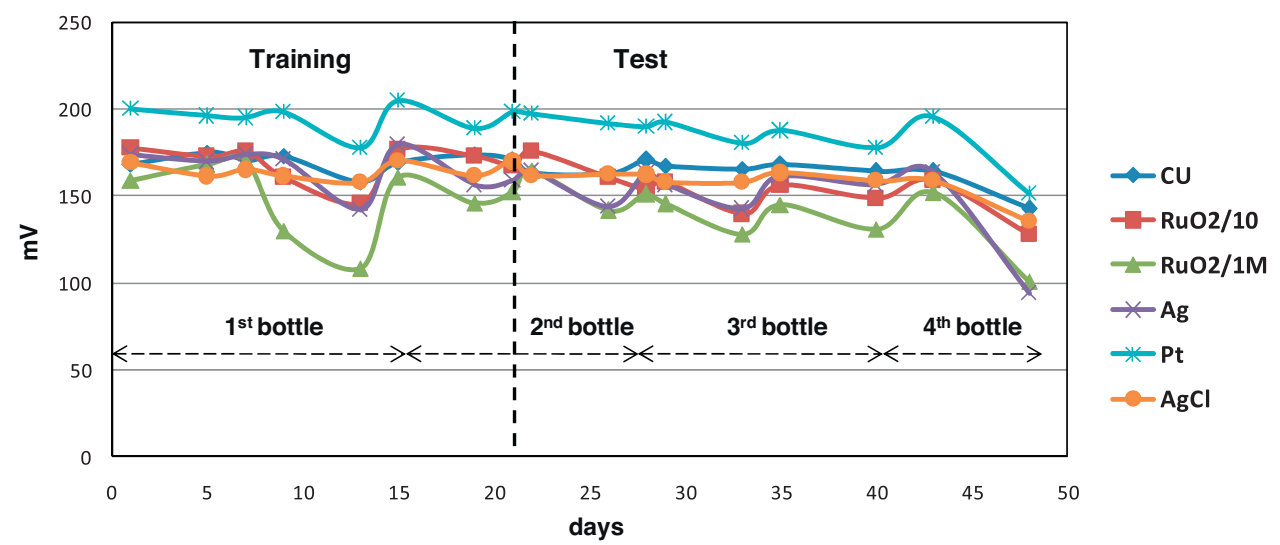

Fig. 4. Response to tap water samples.

response of irrelevant entries [22]. This theory has evolved into a series of neural algorithms for unsupervised learning that are capable of creating stable classes by the presentation of arbitrary input sequences with a fast learning rate [23].

ART network consists of two subsystems (Fig. 5a): A first subsystem to complement the entry code where each input value is doubled by including the value $a_{c}=1-a$; and in this way it avoids the proliferation of categories. The second subsystem is similarity and resonance (Fig. 5b). Whenever the network receives a new input vector $(V)$ the system reacts by activating one of the output nodes $\left(C_{j}\right)$. If the measure does not seem to be already closely assigned to any node, the network creates a new node. The network performance is mainly determined by three parameters $(\rho, \beta$ and $\alpha)$.

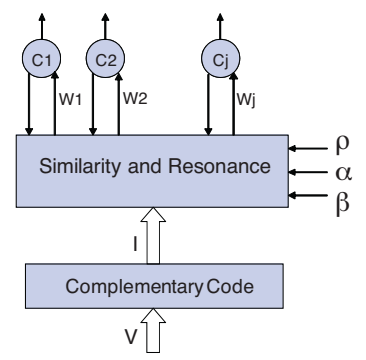

(A) ART

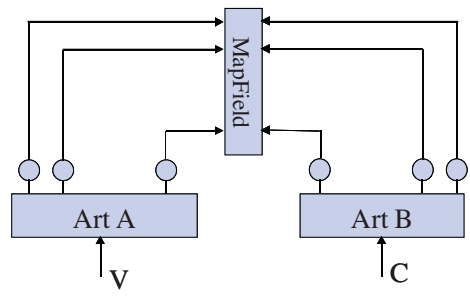

(B) ARTMAP
Fig. 5. The fuzzy ARTMAP architecture.
Monitoring parameter $(\rho)$ determines whether a new measure is part of an existing class or whether another class must be created. The value of this parameter (between 0 and 1 ) determines the level of rigor the algorithm should use when grouping the measures.

The parameter $\beta$ (between 0 and 1) determines the speed of network learning, high values of $\beta$ result in high learning speed while low values causes low learning speed. Additionally it contributes robustness to the classification algorithm, especially when it comes to categorizing data that may have some noise in their values.

The parameter $\alpha$ is called the factor of choice and it enables the system to make the decision in the case that, for a given input, there are two or more categories that can be activated. With a value of $\alpha$ $(>0)$ the output is elected whose weights are modified to the lesser extent.

Fuzzy ARTMAP [24] network consists of two ART-type networks, one that made the training (ART A) and the other the verification (ART B). The connection between the two networks is performed by means of a memory map called the mapfield. A supervised classification is performed through this network. The input data can be either digital or analog in the range between 0 and 1 . In the case of analog values the network is called the Fuzzy ARTMAP.

The Fuzzy ARTMAP networks toolbox, designed by Aaron Garrett, Jacksonville State University, was used on MATLAB ${ }^{\circledR} 2010 \mathrm{~b}$. The Fuzzy ARTMAP was trained in order to obtain the weights, map field and max-min of each input.

The networks were trained with the first eight samples of drinking waters, to give altogether 56 data results of 8 samples by 7 types of water. The selected parameters to train the network were: the 


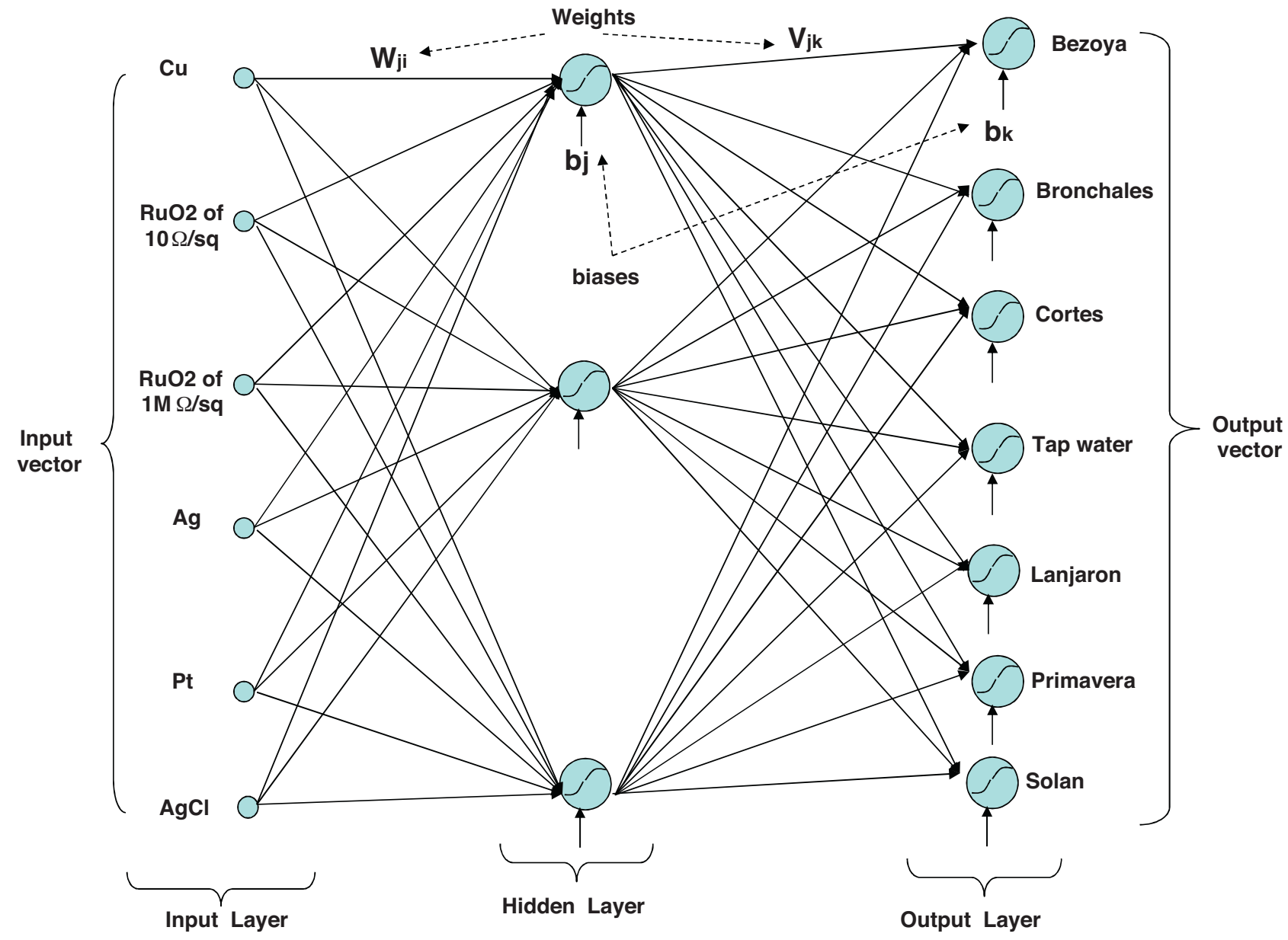

Fig. 6. Multi-layer feed-forward artificial neural network architecture.

vigilance parameter $[\rho]$ was 0.75 , the learning rate $[\beta]$ was 1 and the biasing $\alpha$ was 0.001 .

As a result of the training a $12 \times 16$ weight matrix and a $1 \times 16$ map field were obtained. A maximum and minimum of the input data are also obtained. All these data were used in the firmware of the microcontroller [37].

\subsection{Training the multi-layer feed-forward neural network}

In order to training a multi-layer feed-forward neural network [25], the nprtool GUI (Neural Network Pattern Recognition tool Graphical User Interface) of Matlab ${ }^{\circledR} 2010 \mathrm{~b}$ has been used. The network is a two layer feed-forward type with the default tan-sigmoid transfer functions in both the hidden and output layers. It is called patternnet (Pattern recognition network) in Matlab ${ }^{\circledR}$ (Fig. 6).

Pattern recognition networks are feed-forward networks that can be trained to classify inputs according to target classes. The target data for pattern recognition networks should consist of vectors of all zero values except for a 1 in element $i$, where $i$ is the class they are to represent. Input data are normalized between $[-1,1]$ in order to enhance the neural network algorithm. When an input

Table 2

MSE and Percent Error for training, validation and test of the MLFF network.

\begin{tabular}{lllc}
\hline & Samples & MSE & Error [\%] \\
\hline Training & 40 & 0.0237 & 0 \\
Validation & 8 & 0.0557 & 35.7 \\
Test & 8 & 0.0532 & 12.5 \\
\hline
\end{tabular}

vector of the appropriate category is applied to the network, the corresponding neuron should produce a 1 , and the other neurons should output a 0 .

Tansig (Hyperbolic tangent sigmoid transfer function) activation functions are used for neurons in the hidden nodes and the output nodes, this function is shown in Eq. (1).

$\operatorname{Tansig}(n)=\frac{e^{n}-e^{-n}}{e^{n}+e^{-n}}=\frac{2}{1+e^{-2 n}}-1$

56 samples have been used, 40 of them (70\%) have been used to train, $8(15 \%)$ to valid and $8(15 \%)$ to test. The selection of the samples has been at random using random data division function. The scaled conjugate gradient back-propagation algorithm implemented in MATLAB ${ }^{\circledR}$ is used to train this network. The number of the hidden nodes used was 20.

The values of MSE (Mean Squared Error) and Percent Error for training, validation and test of the MLFF network are shown in Table 2. Fig. 7a shows the confusion matrix. In the confusion matrix the diagonal cells show the number of residue positions that were correctly classified for each structural class. The off-diagonal cells show the number of residue positions that were misclassified. The blue cell shows the total percentage of correctly predicted residues (top number) and the total percentage of incorrectly predicted residues (bottom number). Fig. $7 \mathrm{~b}$ shows the Receiver Operating Characteristic (ROC) curve, a plot of the true positive rate (sensitivity) versus the false positive rate ( 1 - specificity) as the threshold is varied. Fig. 8 shows the histogram error (this shows how the error sizes are distributed, typically most errors are near zero, with very few errors far from that). 


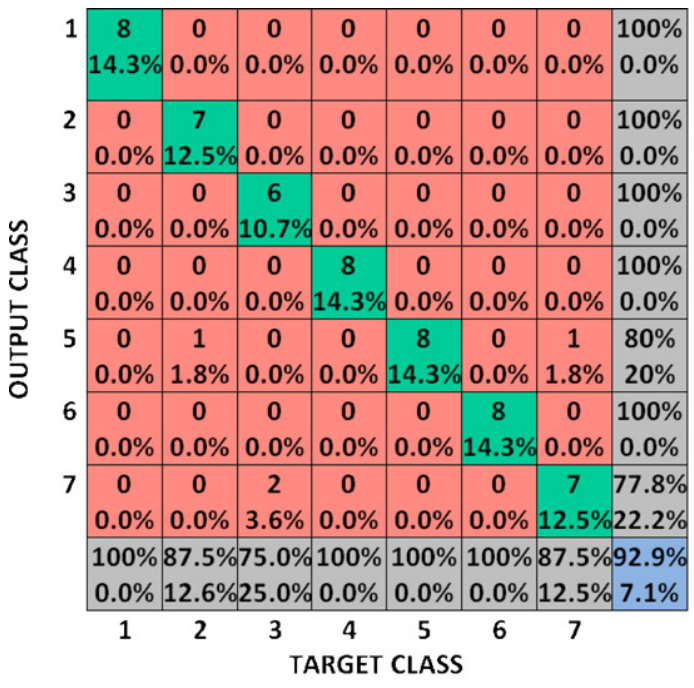

(A) CONFUSION MATRIX

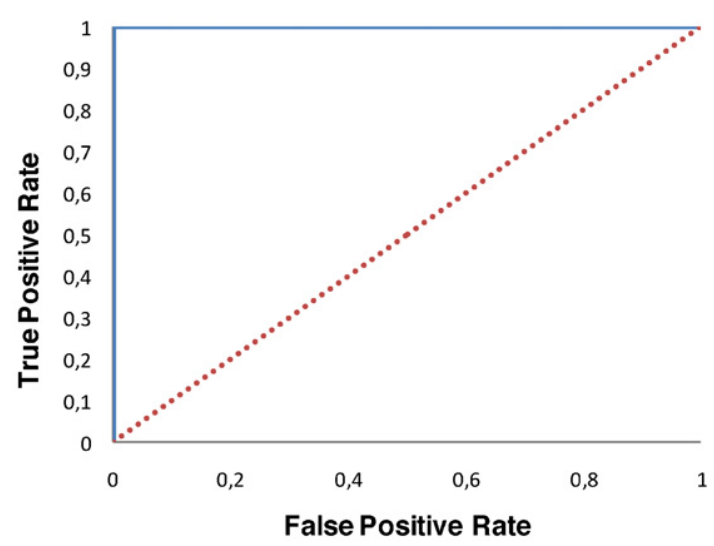

(B) RECEIVER OPERATING CHARACTERISTIC (ROC) CURVE

Fig. 7. Confusion matrix and ROC curve. (a) Confusion matrix and (b) receiver operating characteristic (ROC) curve.

After training two weight matrices were obtained, one of them (from input layer to hidden layer) of $6 \times 2$, the other (from hidden layer to output layer) of $20 \times 7$ as well as a $1 \times 20$ bias matrix (hidden layer) and $1 \times 7$ bias matrix (output layer). A maximum and minimum of input data are also obtained. All these data were used in the firmware of the microcontroller.

\subsection{Training the linear discrimination analysis}

Discriminant analysis (LDA) is a multivariante statistical technique for classifying a set of observations into predefined classes of groups [26]. The objective is to predict group membership of an observation based on a set of input variables known as predictors or training set. The model is built based on a training set for which the classes are known. The technique constructs a set (as many as the number of input variables) of linear functions of the predictors, known as discriminant functions Eq. (2).

$D_{i}=\sum_{k=1}^{p} d_{i k} Z_{k}$

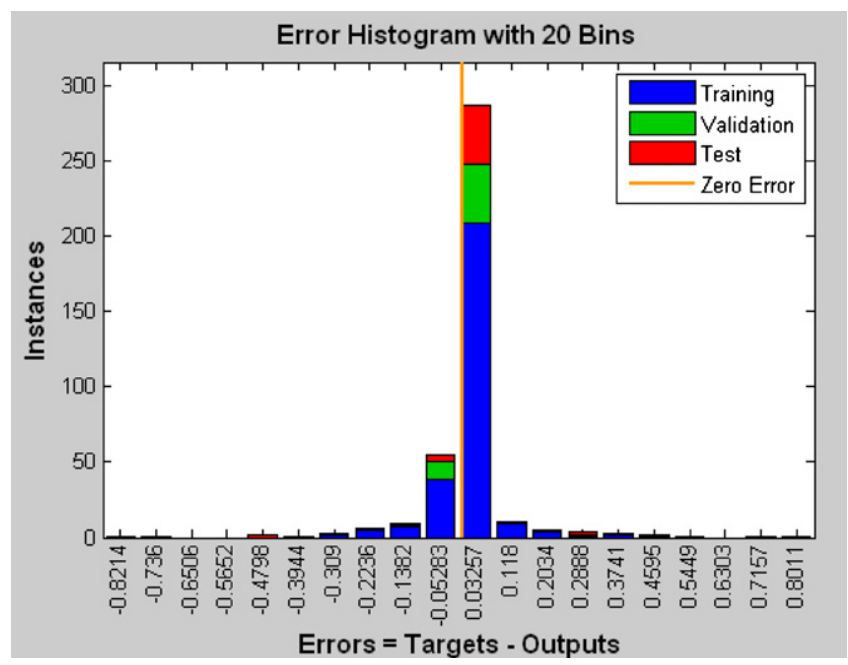

Fig. 8. Error histogram. where the $d_{i k}$ are discriminant coefficients and the $Z_{k}$ are the standardized input variables $I$ created by subtracting the sample means and dividing by the sample standard deviations.

This method maximizes the ratio of between-class variance to the within-class variance (Eq. (3)).

maximize $\frac{\sigma_{\text {between }}^{2}}{\sigma_{\text {within }}^{2}}$

To classify new cases into groups, classification functions are derived. To classify an observation, a score is derived for each group. The score for each group is calculated from Eq. (4).

$C_{i}=c_{i 0}+\sum_{k=1}^{p} c_{i k} I_{k}$

where the $c_{i k}$ are classification function coefficients and the $I_{k}$ are the input variables of new observation. These classification functions are used to predict the class of a new observation with unknown class. With a new observation, all the classification functions are evaluated and the observation is assigned to the class which has the highest value of $C_{i}$.

To determine the discriminant functions, 8 samples by class were used. Among the 56 observations used to fit the model, 54 of them have been classified correctly (96.4286\%). The LDA analysis allowed the selection of a classification model that was based on the 6 predictors. The final model used two discriminant functions with $p$-values less than 0.05 and are statistically significant at a confidence level of $95.0 \%$. In Fig. 9 the scores for the two functions are plotted (explaining $91.53 \%$ and $7.65 \%$ of the total variance, respectively).

The classification function coefficients for classes are used to determine which of the 7 classes any individual sample is most likely to belong to. A $7 \times 7$ matrix classification function coefficients $\left(c_{i k}\right)$ for class have been obtained with the software Statgraphics Centurion XVI.

\section{Implementation of networks in the microcontroller}

\subsection{Introduction}

The embedded system is built around a Microchip PIC18F4550 microcontroller. The PIC18F4550 is a PIC18/8-bit family 


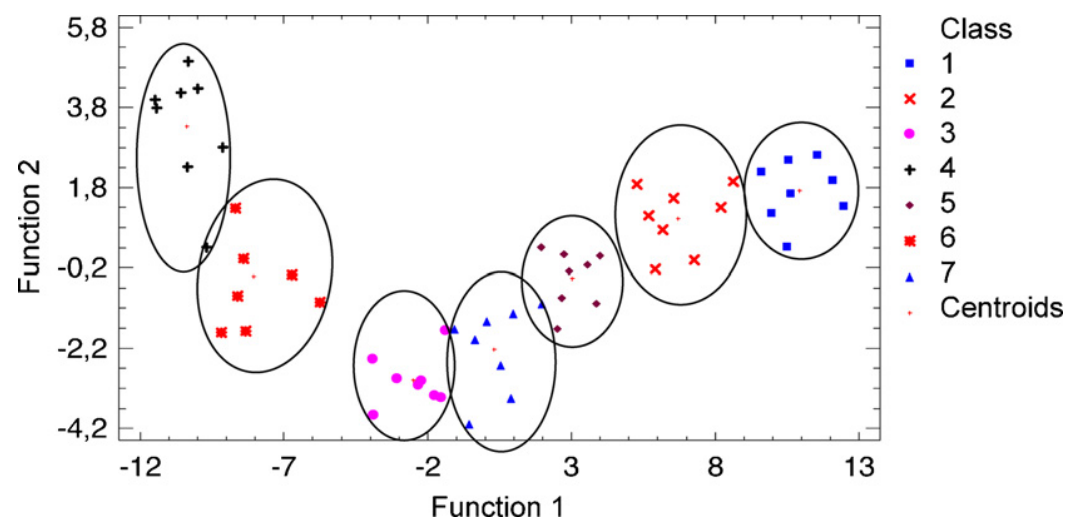

Fig. 9. Plot of discriminant functions.

microcontroller and has $2 \mathrm{~KB}$ of RAM and $32 \mathrm{~KB}$ of reprogrammable flash memory.

The software was coded in C language for the microcontroller and consists of two main routines:

(a) Data acquisition system where the microcontroller reads the data from the A/D converter and processes them in order to obtain the average of each channel.

(b) Implementation of the pattern recognition algorithm [27-35].

\subsection{Implementation of the fuzzy ARTMAP}

In this routine the six input vectors, $I$, are calculated using the 36 channels. The data from 36 channels are acquired and they are also normalized to set their range to $[0,1]$ using the same function of MATLAB ${ }^{\circledR}$, Eq. (5).

$I_{N}=\frac{\left(Y_{\max }-Y_{\min }\right)\left(I-I_{\min }\right)}{\left(I_{\max }-I_{\min }\right)}+Y_{\min }$

where $I$ is the input value, $I_{N}$ is the normalized input value, $Y_{\max }$ and $Y_{\text {min }}$ are maximum and minimum values respectively of interval $[0,1]$ and finally $I_{\max }$ and $I_{\min }$ are the maximum and minimum values of inputs obtained during the training period. The $I_{\max }$ and $I_{\min }$ values can be change by the microcontroller algorithm depending on the new inputs.

In order to preserve the amplitude information the data is complemented, Eq. (6).

$I_{N}=\left(a, a^{c}\right)=\left(a_{1}, \ldots, a_{M}, a_{1}^{c}, \ldots, a_{M}^{c}\right)$

where $a_{i}^{c}=\left(1-a_{i}\right)$.

For each input $I$, the choice function is defined by Eq. (7).

$T_{J}=\frac{\left|I_{N} \Lambda W_{J}\right|}{\alpha+\left|W_{J}\right|}$

where $W_{j}$ are the weights obtained during the training period, operator $\Lambda$ is defined by Eq. (8) and $\alpha$ is called the biasing parameter

$(p \Lambda q)_{i} \equiv \min \left(p_{i}, q_{i}\right)$

And where the norm $|\cdot|$ is defined by Eq. (9).

$|p| \equiv \sum_{i=1}^{M}\left|p_{i}\right|$

The category choice is indexed by $J$, Eq. (10).

$T_{J}=\max \left\{T_{j}: j=1 \ldots N\right\}$
Resonance occurs if the match function of the chosen category meets the vigilance criterion, Eq. (11).

$\frac{\left|I_{N} \Lambda W_{j}\right|}{\left|I_{N}\right|} \geq \rho$

If the match function is less than the vigilance criterion a lesser choice function is selected and the resonance is checked again. Finally if there is no choice function whose match function is greater than the vigilance criterion, the input vector is classified as out of range. If there is resonance then the input vector is classified. The category choice is indexed by $J$. This index $J$ points to the class in the map field. The class is displayed on the LCD panel.

The classification function was implemented onto the microcontroller as shown in Fig. 10.

This routine is coded in the $C$ language and is converted to HEX code using a cross compiler. The HEX file is downloaded into the flash memory of the microcontroller. The fuzzy ARTMAP neural network has been programmed in 12.662 bytes of program memory (39\% ROM) and 1.632 bytes of data memory (79\% RAM).

\subsection{Implementation of the multi-layer feed-forward neural network}

In this routine the six input vectors, $I$, are calculated using the 36 channels. The data from 36 channels are acquired and they are also normalized to set their range to $[-1,1]$ using the same function of MATLAB ${ }^{\circledR}$, Eq. (5).

Weights $\left(W_{j i}\right)$ and biases $\left(B_{j}\right)$ of the trained neural network are obtained from the PC during the training period. Using the input vectors $\left(I_{i N}\right)$, the weights and the biases, the microcontroller calculates the output for each of the twenty hidden nodes by using the following expression Eq. (12).

$y_{j}=\Phi\left(\sum_{i=1}^{N} I_{i N} \cdot W_{j i}+B_{j}\right)$

where: $\Phi$ is the Tansig activation function. The Tansig function can be defined using Eq. (1). $i$ is the input nodes $(i: 1-6) . j$ is the hidden nodes $(j: 1-20)$.

By using this $y_{j}$ data and the weight $\left(V_{k j}\right)$ and biases $\left(B_{k}\right)$ values, the values of output nodes are obtained using the following expression Eq. (13).

$y_{k}=\Psi\left(\sum_{j=1}^{N} y_{j} \cdot V_{k j}+B_{k}\right)$ 


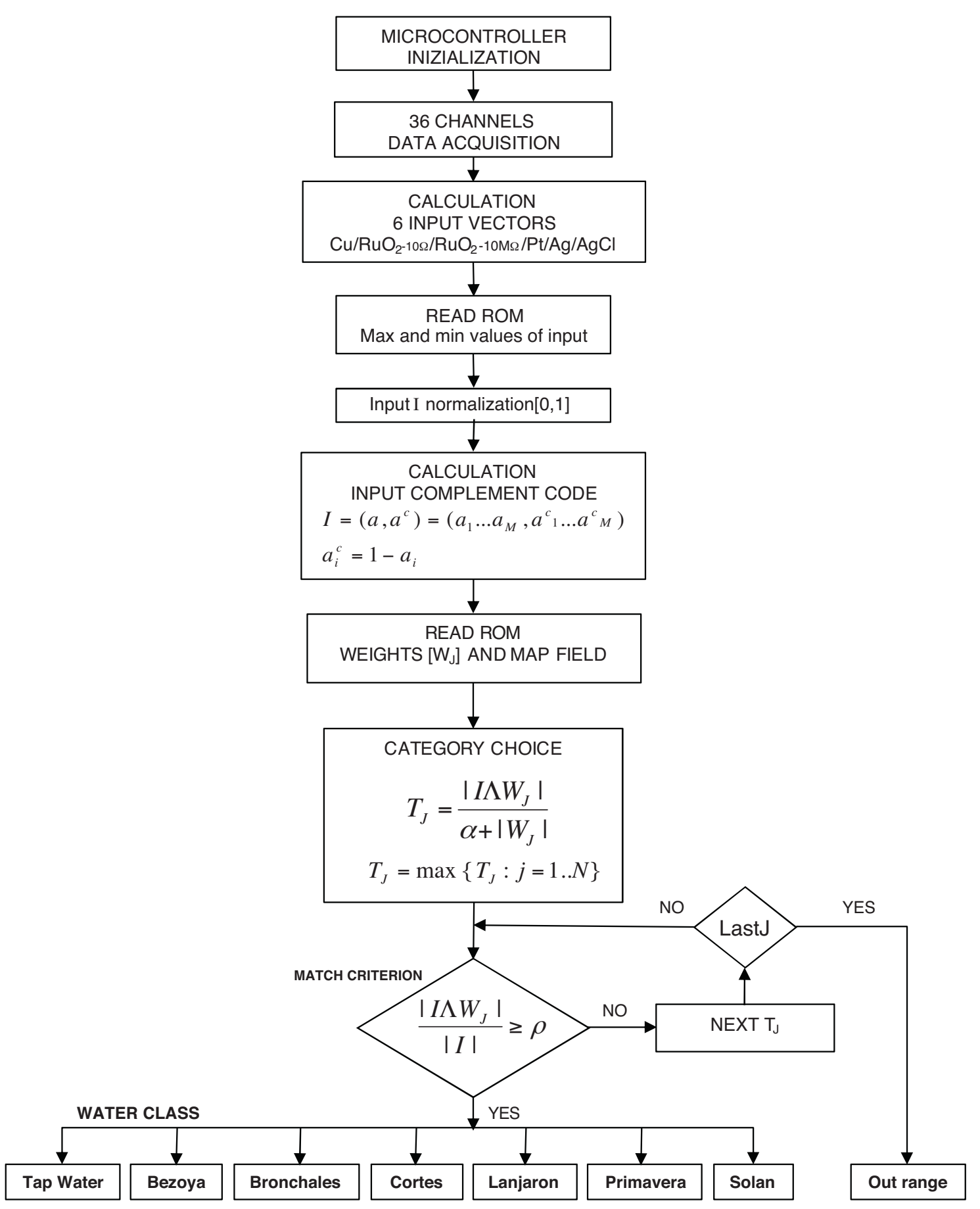

Fig. 10. Fuzzy ARTMAP implementation flowchart in $C$ for microcontroller.

where: $\Psi$ is the Tansig function. $k$ is the output nodes $(k: 1-7) . \mathrm{j}$ is the hidden nodes $(j: 1-20)$ :

Because the output function is a Tansig, the output has a value in the range $[-1,1]$ so they must be made to fit among $[0,1]$ using Eq. (2). Outputs with a value of 1 (or close to it) point to the class of the input vector. The class is displayed on the LCD panel.

This routine is coded in C language and is converted to HEX code using the cross compiler. The HEX file is downloaded into the flash memory of microcontroller. The ANN has been programmed in 12,160 bytes of program memory ( $37 \%$ ROM) and 624 bytes of program memory (30\% RAM).

The whole process can be shown in a flowchart as shown in Fig. 11.

\subsection{Implementation of the discriminant function}

In this routine the six input vectors $\left(I_{k}\right)$ are calculated using the 36 channels. Classification function coefficients $\left(c_{i k}\right)$ of the trained network are obtained from the PC during the training period. Using the input vectors and the classification function coefficients, the microcontroller calculates the class score by using the following expression Eq. (14).

$C_{i}=c_{i 0}+\sum_{k=1}^{p} c_{i k} I_{k}$

where: $c_{i k}$ are the classification function coefficients. $i: 1-7 . k: 1-6$. 


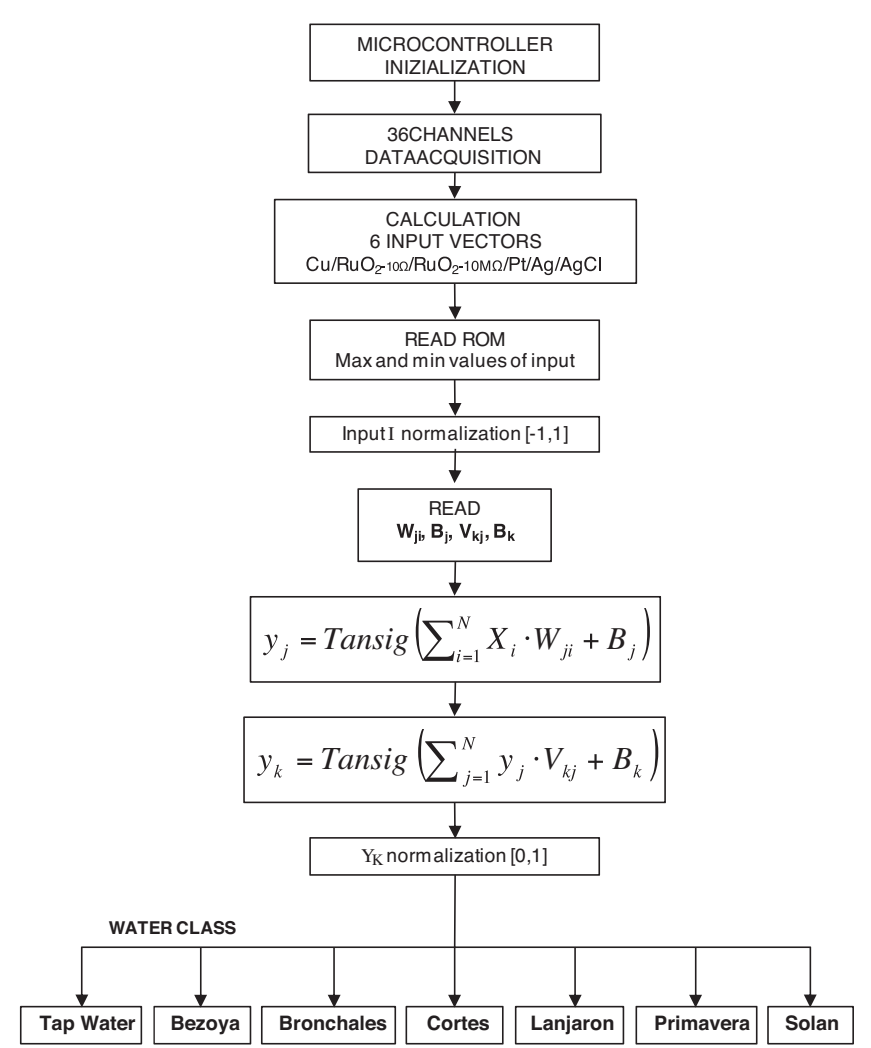

Fig. 11. Multi-layer feed-forward neural network implementation flowchart in C for microcontroller.

The highest value of $C_{i}$ shows the input class. The class is displayed on the LCD panel.

A score is calculated for each observation and each class according to Eq. (14). Each new observation is assigned to whichever class gives the largest value of $C_{i}$. Among the 81 new observations used to validate the model, 67 of them have been classified correctly (82.85\%).

This routine is code in C language and is converted to HEX code using the cross compiler. The HEX file is downloaded into the flash memory of microcontroller. The ANN has been programmed in 9800 bytes of program memory ( $30 \%$ ROM) and 620 bytes of program memory (30\% RAM).

The whole process can be shown in a flowchart as shown in Fig. 12.

\section{Results and discussion}

The artificial neural networks were firstly trained with the array data obtained from several samples of still, sparkling and tap water. Training was done in a PC to obtain the optimum architecture of the

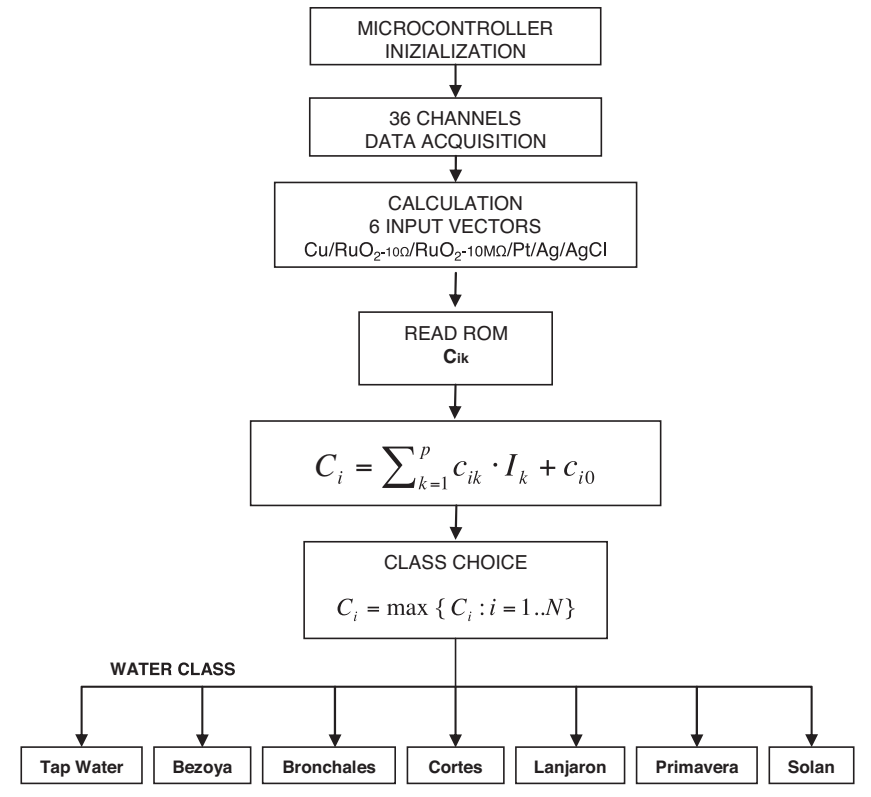

Fig. 12. LDA implementation flowchart in $C$ for microcontroller.

network. Nine samples more were acquired after training and all of them were classified by the microcontroller system. The results are presented in Table 3 where Bezoya is called number 1, Bronchales number 2 , Cortes number 3 , tap water number 4 , Lanjaron number 5 , Primavera number 6 and Solan number 7. The classification of all the samples was the same by the microcontroller and the PC.

Figs. 3 and 4 show the electrodes respond. That signal suffer significant variations, we believe that those variations can be due to two factors; on one hand to the process of ageing of the electrodes and on the other hand to the possible differences of composition of the bottles. These variations were compensated with the reference solution respond and the ability to learn of artificial neural networks [36].

Fig. 13 shows the confusion matrix and the Receiver Operating Characteristic (ROC) for fuzzy ARTMAP, it is observed a recognition rate of $76.2 \%$ is observed in this case. Fig. 14 shows the confusion matrix and the ROC curve for MLFF, it is observed a recognition rate of $76.2 \%$ is observed. Fig. 15 shows the confusion matrix and the ROC curve for LDA, it is observed a recognition rate of $82.5 \%$ is observed in this case.

The most difficult classification is between Bronchales (number 2 ) and Bezoya (number 1 ) because they have very similar concentrations of anions and cations (Table 1). Solan (number 7) and Lanjaron (number 5) are also difficult to classify. The main conclusion from the results of the multi-electrode is that the ions that most affect the results are sulphates, carbonates, chlorides and sodium. The other ions do not significantly affect the response of the

Table 3

Comparison results.

\begin{tabular}{|c|c|c|c|c|c|c|c|c|c|c|c|c|c|c|c|c|c|c|c|c|c|c|}
\hline \multirow[t]{2}{*}{ Sample number } & \multirow[t]{2}{*}{ Bottle number } & \multicolumn{6}{|c|}{ Fuzzy ARTMAP (Classes) } & \multicolumn{8}{|c|}{ MLFF (Classes) } & \multicolumn{7}{|c|}{ LDA (Classes) } \\
\hline & & 1 & 2 & 3 & 4 & 5 & 6 & 7 & 1 & 2 & 3 & 4 & 5 & 6 & 7 & 1 & 2 & 3 & 4 & 5 & 6 & 7 \\
\hline 9th & 2nd & 2 & 5 & 3 & 4 & 5 & 6 & 7 & 1 & 2 & 6 & 4 & 5 & 6 & 7 & 1 & 2 & 7 & 4 & 5 & 6 & 7 \\
\hline 10th & 2nd & 1 & 2 & 3 & 4 & 5 & 6 & 5 & 1 & 2 & 6 & 4 & 5 & 6 & 5 & 1 & 2 & 6 & 4 & 5 & 6 & 7 \\
\hline 11th & $3 r d$ & 1 & 2 & 3 & 4 & 5 & 6 & 7 & 1 & 2 & 3 & 4 & 5 & 6 & 7 & 1 & 2 & 3 & 4 & 5 & 6 & 5 \\
\hline 12th & 3rd & 2 & 2 & 3 & 4 & 5 & 6 & 7 & 1 & 1 & 3 & 4 & 5 & 6 & 5 & 1 & 2 & 3 & 4 & 5 & 6 & 5 \\
\hline 13th & $3 \mathrm{rd}$ & 2 & 2 & 3 & 4 & 5 & 6 & 5 & 1 & 2 & 3 & 4 & 3 & 6 & 3 & 1 & 2 & 3 & 4 & 3 & 6 & 3 \\
\hline 14th & $3 r d$ & 2 & 2 & 3 & 4 & 5 & 3 & 5 & 1 & 1 & 3 & 4 & 5 & 6 & 5 & 1 & 2 & 3 & 4 & 5 & 6 & 5 \\
\hline 15th & 4th & 2 & 2 & 3 & 4 & 7 & 6 & 3 & 1 & 2 & 3 & 4 & 5 & 6 & 3 & 1 & 2 & 3 & 4 & 5 & 6 & 3 \\
\hline 16th & 4th & 1 & 2 & 7 & 4 & 5 & 6 & 5 & 1 & 1 & 5 & 4 & 5 & 6 & 5 & 1 & 2 & 3 & 4 & 5 & 6 & 5 \\
\hline 17th & 4th & 2 & 2 & 3 & 4 & 5 & 6 & 7 & 1 & 1 & 7 & 4 & 5 & 6 & 7 & 1 & 2 & 3 & 6 & 7 & 6 & 7 \\
\hline
\end{tabular}




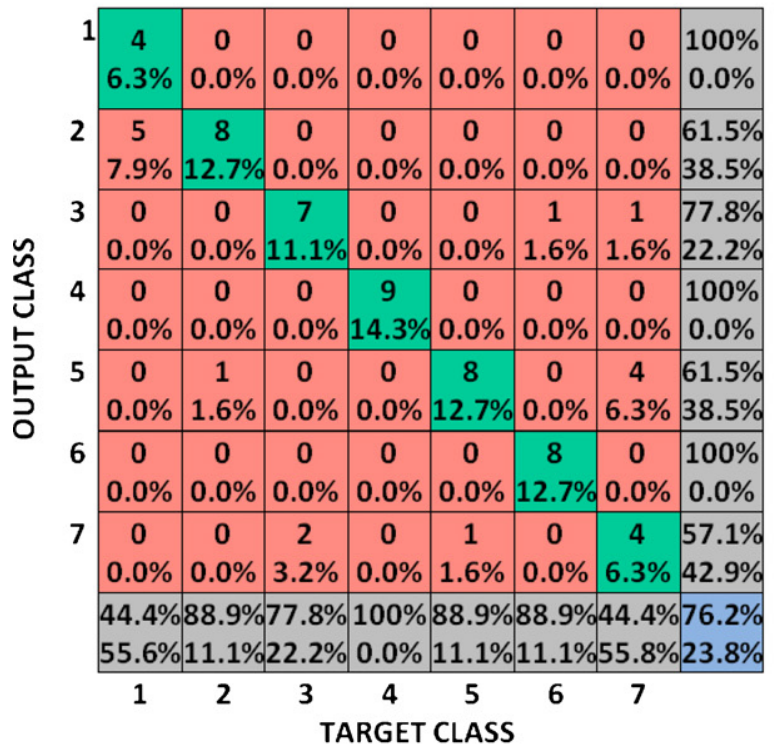

(A) CONFUSION MATRIX

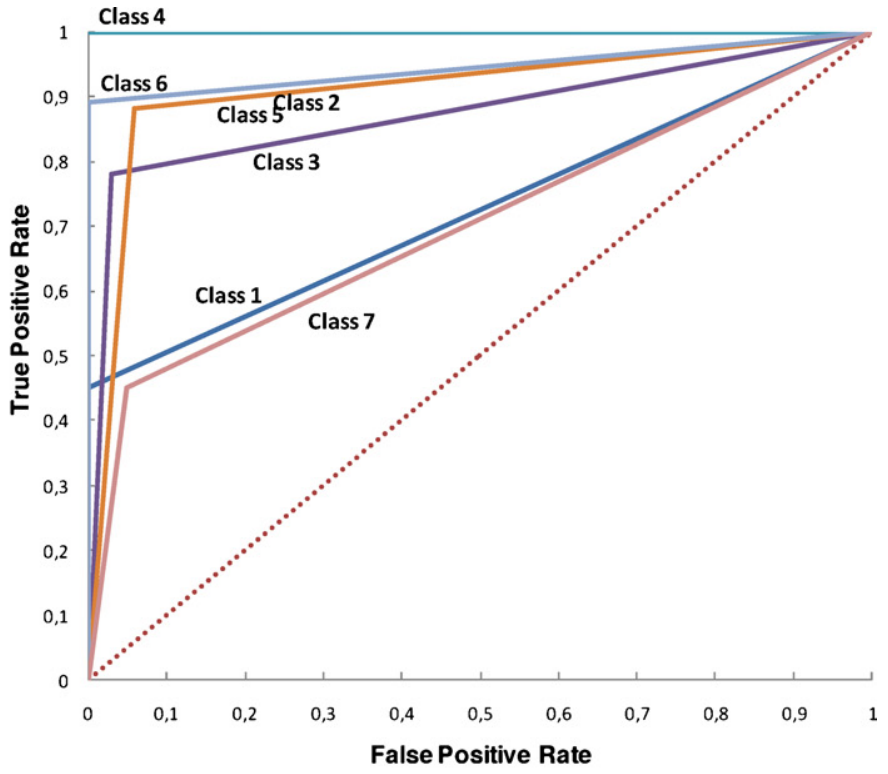

(B) RECEIVER OPERATING CHARACTERISTIC (ROC) CURVE

Fig. 13. Confusion matrix and ROC curve for fuzzy ARTMAP. (a) Confusion matrix and (b) receiver operating characteristic (ROC) curve.

multi-electrode. It is observed that Bezoya and Bronchales are very similar when a PCA analysis of the water (Fig. 16) is made but only taking into account these more significant ions, the same result is achieved as for Solan and Lanjarón. Bearing in mind that the value of PC1 ( $X$ axis) is much higher than PC2 ( $Y$ axis), Lanjarón and Solan are closer than they may appear. The Ion that most significantly determines the outcome of the analysis is $\mathrm{SO}_{4}{ }^{-}$because there is much more difference between tap water and the other waters on this basis.

Recognition rates can be increased when the number of samples is increased. In pattern recognition systems which work on the PC platform it is easy to increase the number of samples for training but when the system works in the microcontroller problems arise with the amount of memory that is used. RAM and ROM size cannot exceed the maximun microcontoller memory in both networks. The amount of program memory used is very similar in both networks but very different amounts of RAM memory are used, with a greater amount in fuzzy ARTMAP than in MLFF or LDA (Table 4).

In the case of increasing the number of samples used for training, the behavior of the networks is different with reference to the amount of used memory. In the MLFF network the memory size only depends on the number of neurons of input, hidden and output layers. This size does not change even though the numbers of samples are increased and Fig. 17 shows how the 20 nodes

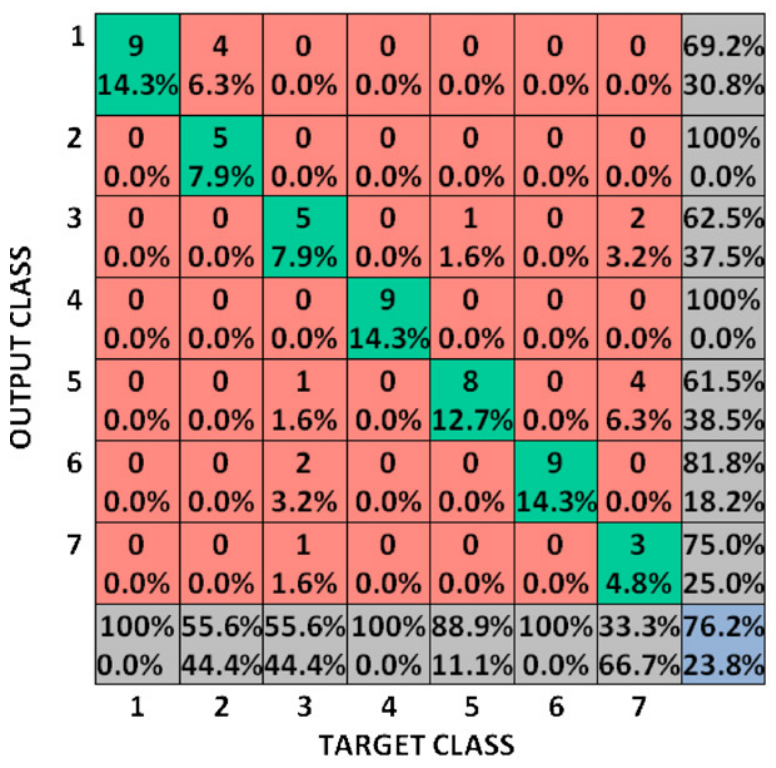

(A) CONFUSION MATRIX

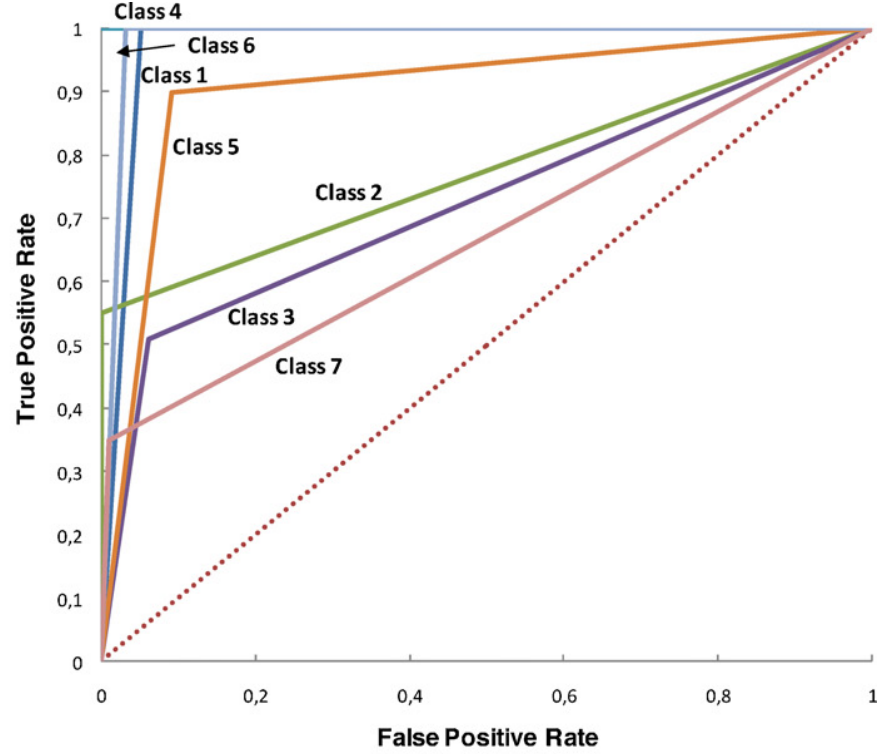

(B) RECEIVER OPERATING CHARACTERISTIC (ROC)

Fig. 14. Confusion matrix and ROC curve for MLFF. (a) Confusion matrix and (b) receiver operating characteristic (ROC) curve. 


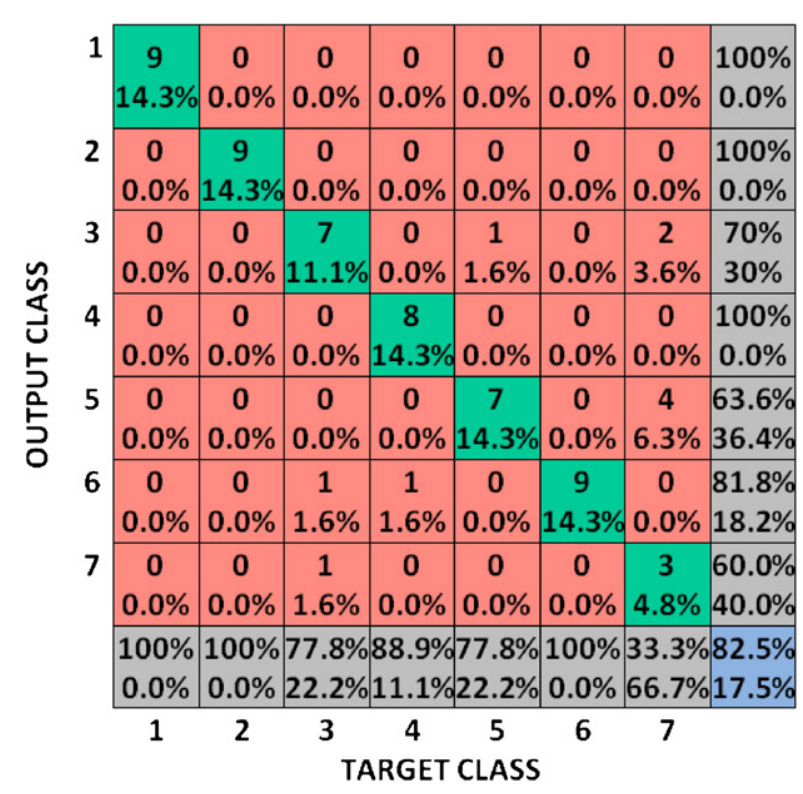

(A) CONFUSION MATRIX

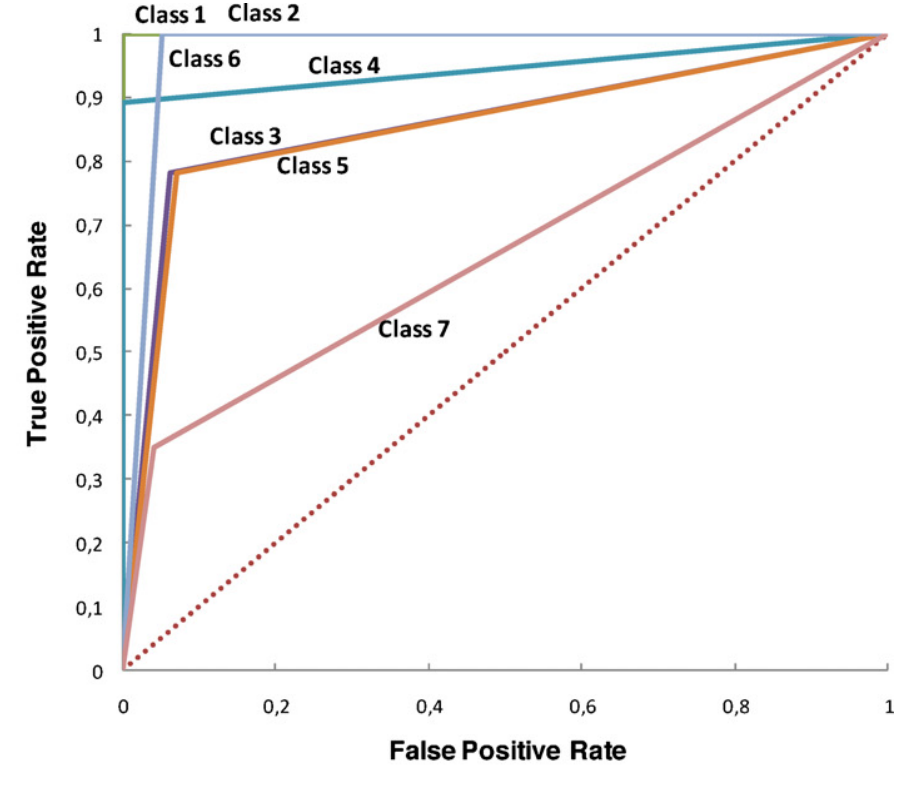

(B) RECEIVER OPERATING CHARACTERISTIC (ROC) CURVE

Fig. 15. Confusion matrix and ROC curve for LDA. (a) Confusion matrix and (b) receiver operating characteristic (ROC) curve.
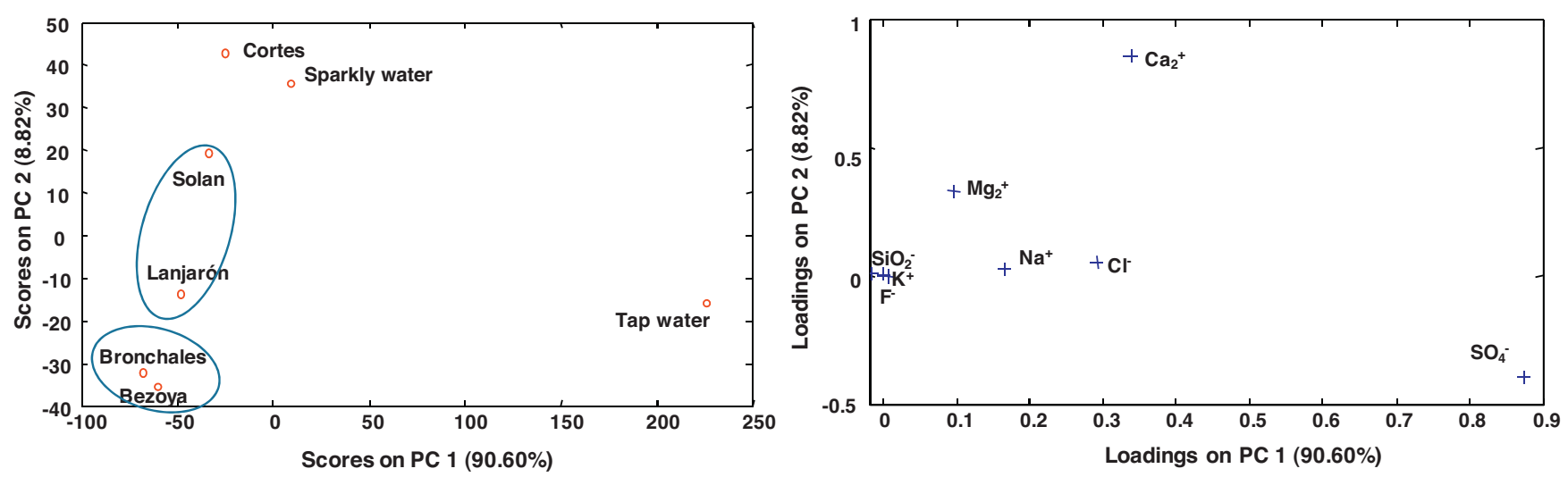

Fig. 16. PCA analysis.

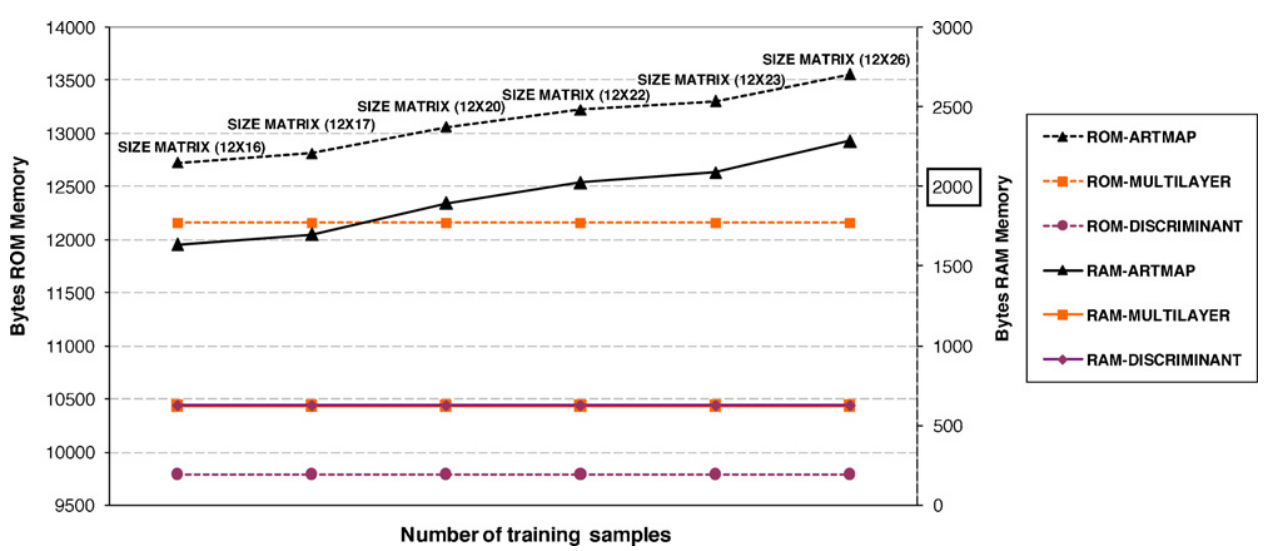

Fig. 17. Size variation of the used memory. 
Table 4

Percentages of RAM and ROM used in the microcontroller for each ANN.

\begin{tabular}{lcr}
\hline \multirow{2}{*}{ Network } & \multicolumn{2}{c}{ Memory used (PIC18F4550) } \\
\cline { 2 - 3 } & ROM & RAM \\
\hline Fuzzy ARTMAP & $39 \%$ & $80 \%$ \\
MLFF & $37 \%$ & $30 \%$ \\
LDA & $30 \%$ & $30 \%$ \\
\hline
\end{tabular}

Table 5

Weight matrix size depending on number of training samples.

\begin{tabular}{cc}
\hline Number of training samples & Weight matrix size \\
\hline 8 & $12 \times 16$ \\
10 & $12 \times 17$ \\
12 & $12 \times 20$ \\
14 & $12 \times 22$ \\
16 & $12 \times 23$ \\
18 & $12 \times 26$ \\
\hline
\end{tabular}

feed-forward memory size is constant. Similarly with LDA, the memory size only depends on the number of constants and Fig. 17 shows how the LDA memory size is also constant. However, in the fuzzy ARTMAP it does not happen this way, the more samples used in training the bigger will be the size of the weight matrix and map field. Table 5 shows this increase. If the matrix size increases, the size of the used memory increases too. Fig. 17 shows this variation for several matrices with different sizes, it is observed that for a $12 \times 22$ weight matrix the used memory exceed $2 \mathrm{~K}$ bytes so, in this case, it will necessary to change the microcontroller. In conclusion, from the point of view of the microcontroller, the use of Feed-Forward network is more efficient than the fuzzy ARTMAP.

\section{Conclusion}

A microcontroller-based electronic tongue system, capable of discriminating between drinking water samples has been successfully developed. An $82.5 \%$ recognition rate has been achieved for the samples tested. This intelligent system may find application in the area of water quality monitoring.

Pattern recognition algorithms have been applied to the classification. The main memory requirement for the algorithms can be minimized sufficiently to fit in the limited memory space of a microcontroller. MLFF networks need many more training cycles $[17,20]$ than fuzzy ARTMAP and LDA. The algorithm which used the most memory of the microcontroller was the Fuzzy ARTMAP. MLFF and LDA used similar amounts of RAM memory but MLFF needs more program memory. Thus, the best pattern recognition algorithm to be implemented on a microcontroller is LDA.

At present we are working with three research lines based on this work; honey classification, meal classification and chemical classification in a waste water depuration plant. Moreover, we are developing new electrodes as well as improvements in electrode stability as major topics for future work.

\section{Acknowledgements}

Dr. E. Garcia gratefully acknowledges financial support (grant BEST/2010/138) from the Generalitat Valenciana and (grant PAID00-10) from the Universidad Politécnica de Valencia during his stay at the University of Southampton. We also thank MICINN (MAT2009-14564-C04-02).

\section{References}

[1] F. Winquist, C. Krantz-Rülker, I. Lunström, Electronic tongues and combinations of artificial senses, Sensors Update 11 (2002) 279-306.
[2] A. Legin, A. Rudnitskaya, Y. Vlasov, C. Di Natale, F. Davide, A. D’Amico, Tasting of beverages using an electronic tongue, Sensors and Actuators B 44 (1997) 291-296.

[3] A. Arrieta, C. Apetrei, M.L. Rodríguez-Méndez, J.A. de Saja, Voltammetric sensor array based on conducting polymer-modified electrodes for the discrimination of liquids, Electrochimica Acta 49 (2004) 4543-4551.

[4] G. Pioggia, F. Di Francesco, A. Marchetti, M. Ferro, A. Ahluwalia, A composite sensor array impedentiometric electronic tongue: part I. Characterization, Biosensors and Bioelectronics 22 (2007) 2618-2623.

[5] H. Sakai, S. Liyama, K. Toko, Evaluation of water quality and pollution using multichannel sensors, Sensors and Actuators B 66 (2000) 251-255.

[6] J. Gallardo, S. Alegret, M. del Valle, Application of a potentiometric electronic tongue as a classification tool in food analysis, Talanta 66 (2005) 1303-1309.

[7] G. Verrelli, L. Francioso, R. Paolesse, P. Siciliano, C. Di Natale, A. D'Amico, A. Logrieco, Development of silicon-based potentiometric sensors: towards a miniaturized electronic tongue, Sensors and Actuators B 123 (2007) 191-197.

[8] L. Lvova, E. Martinelli, E. Mazzone, A. Pede, R. Paolesse, C. Di Natale, A. D'Amico, Electronic tongue based on an array of metallic potentiometric sensors, Talanta 70 (2006) 833-839.

[9] J. Soto, R.H. Labrador, M.D. Marcos, R. Martínez-Máñez, C. Coll, E. García-Breijo, L. Gil, A model for the assessment of interfering processes in Faradic electrodes, Sensors and Actuators 142 (2008) 56-60.

[10] R. Martínez-Máñez, J. Soto, E. García-Breijo, L. Gil, J. Ibañez, E. Gadea, A multisensor in thick-film technology for water quality control, Sensors and Actuators A 120 (2005) 589-595.

[11] B.A. Botre, D.C. Gharpure, A.D. Shaligram, Embedded electronics nose and supporting software tool for its parameter optimization, Sensors and Actuators B 146 (2010) 453-459.

[12] A.S. Abdul Rahman, M.M. Sim Yap, A.Y.Md. Shakaff, M.N. Ahman, Z. Dahari, Z. Ismail, M.S. Hitam, A microcontroller-based taste sensing system for the verification of Eurycomalongifolia, Sensors and Actuators B 101 (2004) 191-198.

[13] R.E. Shaffer, S.L. Rose-Pehrsson, R.A. McGill, A comparison study of chemical sensor array pattern recognition algorithms, Analytica Chimica Acta 384 (1999) 305-317.

[14] D. Svozil, V. Kvasnicka, J. Pospichal, Introduction to multi-layer feed-forward neural networks, Chemometrics and Intelligent Laboratory Systems. 39 (1997) 43-62.

[15] R. Martínez-Máñez, J. Soto, E. Garcia-Breijo, L. Gil, J. Ibáñez, E. Llobet, An electronic tongue design for the qualitative analysis of natural waters, Sensors and Actuators B 104 (2005) 302-307.

[16] G.J. McLachlan, Discriminant Analysis and Statistical Pattern Recognition, Wiley Interscience, New York, 2004

[17] K. Iizuka, T. Aishima, Differentiation of soy sauce by pattern recognition analysis of mid-and near-IR spectra, Journal of Food Composition and Analysis 12 (1999) 197-209.

[18] E. Llobet, E.L. Hines, J.W. Gardner, P.N. Bartlett, T.T. Mottram, Fuzzy ARTMAP based electronic nose data analysis, Sensors and Actuators B 61 (1999) 183-190.

[19] C. Di Natale, A. Macagnano, F. Davide, A. D’Amico, A. Legin, Y. Vlasov, A. Rudnitskaya, B. Selezenev, Multicomponent analysis on polluted waters by means of an electronic tongue, Sensors and Actuators 44 (1997) 423-428.

[20] L. Gil, E. Garcia-Breijo, J. Ibañez, R.H. Labrador, E. Llobet, R. Mantínez-Mañez, J. Soto, Electronic tongue for qualitative analysis of aqueous solutions of salts using thick-film technology and metal electrodes, Sensor 6 (2006) 1128-1138.

[21] B. Martín-del-Brío, A. Bono-Nuez, N. Medrano-Marqués, Self-organizing maps for embedded processor selection, Microprocessors and Microsystems 29 (2005) 307-315

[22] G.A. Carpenter, S. Gossberg, ART2: self-organization of stable category recognition codes for analog input patterns, Applied Optics 26 (1987) 4919-4930.

[23] G.A. Carpenter, S. Gossberg, N. Markuzon, J. Reynolds, D. Rosen, Artmap Fuzzy, A neural network architecture for incremental supervised learning of analog multidimensional maps, IEEE Transactions on Neural Networks 3 (1992) 698-713.

[24] G.A. Carpenter, S. Grossberg, Fuzzy, ARTMAP: a synthesis of neural networks and fuzzy logic for supervised categorization and nonstationary prediction, in: R.R. Yager, L.A. Zadeh (Eds.), Fuzzy Sets, Neural Networks and Soft Computing, Van Nostrand-Reinhold/New York, 1994, pp. 126-165.

[25] D. Svozil, V. Kvasnicka, J. Pospichal, Introduction to multi-layer feed-forward neural networks, Chemometrics and Intelligent Laboratory Systems 39 (1997) 43-62.

[26] K. Fukunaga, Introduction to Statistical Pattern Recognition, Academic Press Professional, San Diego, 1990.

[27] S.S. Chowdhury, B. Tudu, R. Bandyopadhyay, N. Bhattacharyya, Portable electronic nose system for aroma classification of black tea, in: 2008 IEEE Region-10 Colloquium and the Third ICIIS, 2008, pp. 1-5.

[28] N.J. Cotton, B.M. Wilamowski, A neural network implementation on an inexpensive eight bit microcontroller, in: INES2008-12th International Conference on Intelligent Engineering Systems, 2008, pp. 109-114.

[29] B. Tudu, N. Bhattacharyya, R. Bandyopadhyay, Normalization techniques for gas sensor array as applied to classification for black tea, International Journal on Smart Sensing and Intelligent Systems (2009) 176-189.

[30] P. Neelamengam, A. Rajendran, Neural network based density measurement, Bulgarian Journal of Physics 31 (2004) 163-169.

[31] S. Bashyal, G.K. Venayagamoorthy, B. Paudel, Embedded neural network for fire classification using an array of gas sensors, in: SAS 2008-IEEE Sensors Applications Symposium, 2008, pp. 146-148. 
[32] J. Tang, Y. Zhang, X. Tang, J. Liu, Nonlinearity correction of the thermocouple based on neural network, in: 2009 WRI Global Congress on Intelligent Systems 3, 2009, pp. 28-32.

[33] M.S. Cosio, D. Ballabio, S. Benedetti, C. Gigliotti, Evaluation of different storage conditions of extra virgin olive oils with an innovative recognition tool built by means of electronic nose and electronic tongue, Food Chemistry 101 (2007) 485-491.

[34] A.M. Peres, L.G. Dias, T.P. Barcelos, J. SáMorais, A.A.S.C. Machado, An electronic tongue for juice level evaluation in non-alcoholic beverages, Procedia Chemistry 1 (2009) 1023-1026.

[35] L.A. Dias, A.M. Peres, A.C.A. Veloso, F.S. Reis, M. Vilas-Boas, A.A.S.C. Machado, An electronic tongue taste evaluation: identification of goat milk adulteration with bovine milk, Sensors and Actuators B 136 (2009) 209-217.

[36] J.K. Atkinson, M. Glanc, P. Boltryk, M. Sophocleous, E. Garcia-Breijo, An investigation into the effect of fabrication parameter variation on the characteristics of screen-printed thick-film silver/silver chloride reference electrodes, Microelectronics International 28 (2) (2011) 49-52.

[37] E. Garcia-Breijo, J. Atkinson, J. Garrigues, L. Gil, J. Ibañez, M. Glanc, C. Olguin, An electronic tongue for monitoring drinking waters using a fuzzy ARTMAP neural network implemented on a microcontroller, in: 2011 IEEE International Symposium on Industrial Electronics, 2011, pp. 1270-1275, doi:10.1109/ISIE.2011.5984341.

\section{Biographies}

Eduardo García-Breijo received his M.Sc. degree in Electronic Engineering at Universitat de València (Spain) in 1997, and received his Ph.D. degree in 2004 at Universidad Politécnica de Valencia (UPV). He is an assistant professor of Electronics Engineering Department of the Universidad Politécnica de Valencia (UPV). He is a member of the Institute of Molecular Recognition and Technological Development (IDM). His main areas of interest are the development of multisensors in thick-film technology, pattern recognition and microcontrollers.

John Atkinson began his career in 1970 as a Merchant Navy, Radio and Electronics Officer. In 1981 he graduated from the University of Essex with a first class honours degree in Computer Engineering. He was subsequently a Senior Engineer working on pattern recognition systems for speech, vision and hand-written computer input with Quest Automation Research Limited, prior to taking up the post of Lecture in Electronics and Computer Science at the University of Southampton from where he obtaining his Ph.D. in 1999. He is currently a Reader in the School of Engineering Sciences and Editor of the journal Microelectronics International. His research interests include thick-film technology, electronic instrumentation and sensors.

Luis Gil Sánchez received his M.Sc. degree in Electronic Engineering at Universitat de Valencia (Spain) in 1998, and received his Ph.D. in 2007 at Universidad Politécnica de Valencia (UPV). He is assistant professor of Electronics Engineering Department of the Universidad Politécnica de Valencia (UPV). He is a member of the Institute of Molecular Recognition and Technological Development (IDM). His main areas of interest are the chemical sensors, instrumentation systems and pattern recognition for electronic tongues.

Rafael Masot Peris obtained his M.Sc. degree in Physics at Universidad de València, Spain (UV) in 1991 and his M.E. degree in electronic engineering at UV, 1996 and received his Ph.D. in 2010 at Universidad Politécnica de Valencia (UPV). He is assistant professor of the Electronics Engineering Department of the Universidad Politécnica de Valencia (UPV). He is a member of the Institute of Molecular Recognition and Technological Development (IDM). His research interest includes modeling EIS, multivariable analysis and design of electronic systems for electrochemical measurements techniques.

Javier Ibáñez Civera is Matrisse in Power Electronic and Control at Universite Pierre et Marie Curie (Paris VI) in 1994 and received his Ph.D. in 2009 at Universidad Politécnica de Valencia (UPV). He is assistant professor of Electronic Technology in the Electronic Engineering Department of the Universidad Politécnica de Valencia (UPV). He is a member of the Institute of Molecular Recognition and Technological Development (IDM). His main areas of interest are water organic contamination devices.

José Garrigues Baixauli obtained his M.Sc. degree in Industrial Engineering at Universidad Politécnica de Valencia in 1983. He is assistant professor of Electronic Technology in the Electronic Engineering Department of the Universidad Politécnica de Valencia (UPV). He is a member of the Institute of Molecular Recognition and Technological Development (IDM). His main areas of interest are Artificial Neural Networks applications (ANN) by microcontrollers. Nowadays he is developing his Thesis in these applications.

Monika Glanc-Gostkiewicz obtained her M.Sc. in Soil Science in 2002 from Nicolaus Copernicus University in Poland. After graduating she moved to United Kingdom where she studied at the University of Portsmouth and received a PGCE qualification. She subsequently taught for three years in secondary schools in Hampshire. She is currently working on her Ph.D. under the supervision of Dr John Atkinson.

Nicolás Laguarda Miro was graduated in Environmental Sciences from the Universidad Politécnica de Valencia in 2000. He received his Ph.D. from the same university in 2005. Nicolás is nowadays a member of the Instituto del Agua y del Medio Ambiente as well as a teacher of environmental subjects in the ETSID (Escuela Técnica Superior de Ingeniería del Diseño) at UPV. His main areas of interest are the environmental quality control, environmental impacts assessment and technology applications to these areas.

Cristian Olguín received the Engineering degree in Automation and Industrial Electronics at the Universidad Politecnica de Valencia in 2010 with a degree thesis entitled: Design of an electronic system for the implementation of the electrochemical measurement techniques: cyclic voltammetry, impedance spectroscopy and potentiometry. And now he is a doctoral student at Universidad Politécnica de Valencia. His research focuses on the electrochemical sensors. 